\title{
Present Progress of Plasma Transport Study on HL-2A
}

\author{
Xuantong DING, Weiwen XIAO, Xiaolan ZOU ${ }^{1)}$, Hongjuan SUN, Yi LIU, Lianghua YAO, \\ Jun RAO, Beibing FENG, Zetian LIU, Yan ZHOU Qingwei YANG, Longwen YAN, \\ Jiaqi DONG, Xuru DUAN and HL-2A team \\ Southwestern Institute of Physics, P.O. Box 432, Chengdu, China \\ 1)Association Euratom-CEA, CEA/DSM/IRFM, CEA/Cadarache, 13018 St Paul-lez-Durance, France
}

(Received 17 February 2009 / Accepted 22 July 2009)

\begin{abstract}
The recent experiments of HL-2A tokamak have been focused on studying the physics of turbulence and transport. A spontaneous particle transport barrier has been observed in Ohmic discharges without any external momentum input. The barrier was evidenced by density perturbation study using modulated supersonic molecular beam injection (SMBI) and microwave reflectometry. The new features of the non-local transport effect induced with SMBI have been analyzed. The suppression of $m / n=2 / 1$ tearing modes may be sustained by ECRH modulation at a frequency about $10 \mathrm{~Hz}$. Continuous confinement improvement was observed after the mode suppression.
\end{abstract}

(c) 2010 The Japan Society of Plasma Science and Nuclear Fusion Research

Keywords: particle transport barrier, non-local transport, supersonic molecular beam injection, electron cyclotron heating

DOI: $10.1585 /$ pfr.5.S1013

\section{Introduction}

The understanding of transport physics and plasma confinement is an important subject for the design of future fusion reactors, especially the physics for particle transport [1-4], electron heat transport [5-8] and the internal transport barrier formation [9-11]. The recent experiments of HL-2A tokamak ( $R=1.65 \mathrm{~m}, a=0.4 \mathrm{~m})$ have been focused on studying the physics of turbulence, transport, MHD instabilities and energetic electron dynamics. This paper presents some new experimental results for transport on HL-2A, including the spontaneous particle transport barrier, the non-local phenomena triggered by SMBI and the confinement features during modulated ECRH.

\section{Present Status of the HL-2A Toka- mak}

The main operation parameters of the device are as follows: the toroidal magnetic field is $1.2-2.7 \mathrm{~T}$, the plasma current is $150-450 \mathrm{kA}$ and the plasma density is $(1-6) \times 10^{19} \mathrm{~m}^{-3}$. ECRH system with four $68 \mathrm{GHz} /$ $500 \mathrm{~kW} / 1 \mathrm{~s}$ gyrotrons has been built up in HL-2A. The ECRH power with both fundamental O-mode and second harmonic X-mode up to $2 \mathrm{MW}$ is injected from the low field side of the device. The deposit position of ECRH is determined by the toroidal magnetic field. When toroidal magnetic field varies from $2.43 \mathrm{~T}$ to $2.2 \mathrm{~T}$ for O-mode, the resonance point can be replaced from the plasma core to the point at $r=16 \mathrm{~cm}$. The wave energy deposits in a range of $3 \mathrm{~cm}$. The efficiency of the transmission system is more than $80 \%$, so at least $1.6 \mathrm{MW}$ power can be injected into

author's e-mail:dingxt@swip.ac.cn plasmas. The maximal electron and ion temperatures are $5 \mathrm{keV}$ and $1.5 \mathrm{keV}$ during $2 \mathrm{MW}$ ECRH respectively.

The supersonic molecular beam injection (SMBI) system has been improved with the modulation frequency reaching $50 \mathrm{~Hz}$. Particles of the gas are accelerated by imposed pressure through the nozzle to get into the vacuum chamber of the tokamak. It is an attempt to enhance the penetration depth and fuelling effect. The penetration depth is very important to trigger the non-local transport phenomena. $\mathrm{H} \alpha$ intensity profile measured by $\mathrm{H} \alpha$ detector array and the density profiles measured by microwave reflectometry indicate that the penetration depth of the SMBI is more than $\rho=0.7$. The SMBI injection has deeper penetration and better locality than conventional gas puffingthe system is not only an advanced technology for fuelling, but also a powerful tool (combined with microwave reflectometer or ECE) for particle as well as electron thermal transport studies.

The device is equipped with extensive and advanced diagnostics for transport study, including ECE and microwave reflectometry with high spatial and temporal resolution. There are also the Thomson scattering for electron temperature measurement, the 8 channel HCN laser interferometer for density profile, the five soft x-ray arrays for MHD study and the Doppler reflectometry for plasma rotation and core turbulence measurements.

\section{Experimental Results}

\subsection{Spontaneous internal particle transport barrier \\ On HL-2A tokamak, spontaneous particle transport}


barriers were observed in purely Ohmic heating plasmas without any external momentum input. The internal particle transport barriers (pITBs) were found to be formed when the line averaged electron density increases to a critical value in HL-2A experiments. Figure 1 shows the temporal evolution of the density profile measured by a broadband O-mode reflectometer for a representative discharge with a transition to pITB formation. The pITB developed gradually around $r=29 \mathrm{~cm}$. The critical lineaveraged electron density corresponding to this transition is $n_{\mathrm{c}}=2.2 \times 10^{19} \mathrm{~m}^{-3}$. The width of the barrier is $1-2 \mathrm{~cm}$. There is a drastic change in the density gradient through the barrier: $L_{\mathrm{n}} \sim 10 \mathrm{~cm}$ at the barrier, $L_{\mathrm{n}} \sim 50 \mathrm{~cm}$ for $r=20-28 \mathrm{~cm}$ and $L_{\mathrm{n}} \sim 25 \mathrm{~cm}$ for $r=30-36 \mathrm{~cm}$.

To study the transport features of the pITB, the density modulation generated by SMBI has been employed. In cylindrical geometry the particle transport equation is simply given by:

$$
\frac{\partial n_{e}}{\partial t}=\frac{1}{r} \frac{\partial}{\partial r}\left[r D \frac{\partial n_{e}}{\partial r}+r V n_{e}\right]+S(r, t)
$$

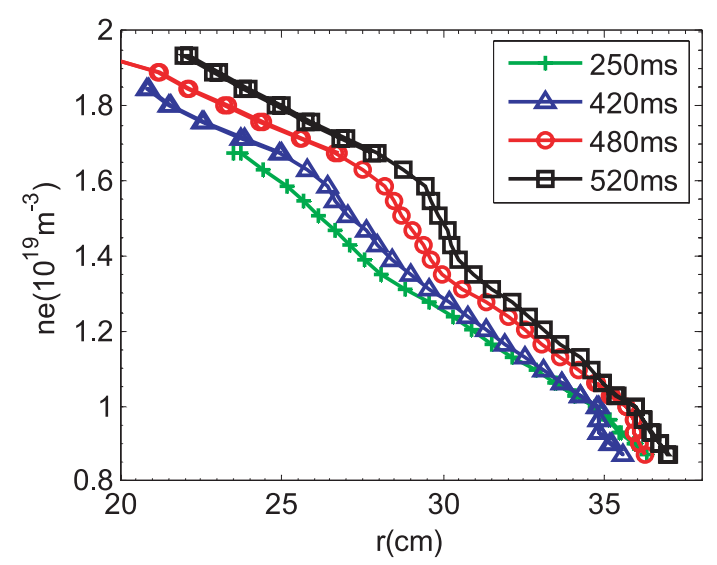

Fig. 1 Temporal evolution of the density profile at selected moments: before the appearance of the barrier $(250 \mathrm{~ms})$; at the beginning of the appearance of the barrier $(420 \mathrm{~ms})$; strong barrier $(480 \mathrm{~ms})$; just after a particle pulse injection by SMBI $(520 \mathrm{~ms})$.

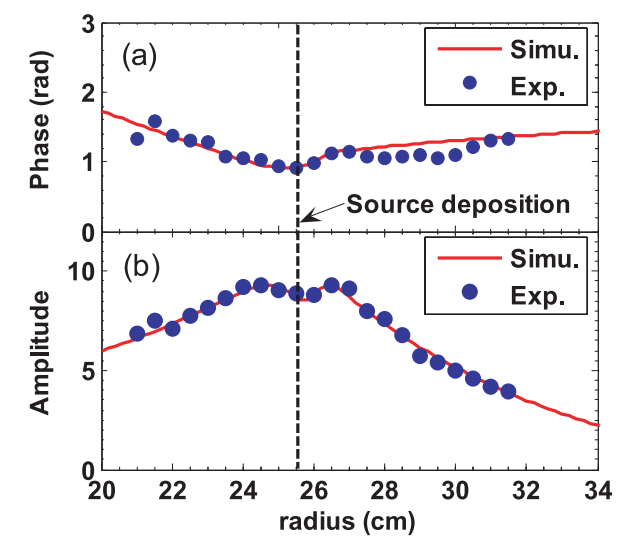

with the following control parameters, the diffusivity $D$ and the convective velocity $V . S$ is the particle source. The modulation frequency and the pulse duration of the SMBI are $9.6 \mathrm{~Hz}$ and about $6 \mathrm{~ms}$, respectively, while the gas pressure is $1.3 \mathrm{MPa}$ in the experiments. The simulation with an analytical model [12] for particle transport was carried out to characterize this barrier quantitatively. The relative shape of the particle source is given by a Gaussiandistribution in the analytical model and its location is measured from a $\mathrm{H}$-alpha array. The measurement range is divided to three areas and one of them is in the barrier. For the simulation, we assume that the transport coefficients are constant in each area. Then the density $n_{\mathrm{e}}(r, t)$ can be calculated from the formula (33) in the Ref. [12] with the assumed transport coefficients. After FFT, we can obtain the amplitude and phase of the density perturbation to match the data of the experiment. The phase and the amplitude of the 1st harmonic of the modulated density are displayed in Figs. 2 (a) and (b), where the solid lines and closed circles are the simulation and experimental results, respectively. The model diffusivity $D$ and convective velocity $V$ employed in the simulation are presented in Fig. 2(c): $D_{1}=0.1 \mathrm{~m}^{2} / \mathrm{s}, V_{1}=1.0 \mathrm{~m} / \mathrm{s}$ in the domain I $\left(r<x_{1}\right) ; D_{2}=0.045 \mathrm{~m}^{2} / \mathrm{s}, V_{2}=-2.7 \mathrm{~m} / \mathrm{s}$ in the domain II $\left(x_{1}<r<x_{2}\right)$, and $D_{3}=0.5 \mathrm{~m}^{2} / \mathrm{s}, V_{3}=6.0 \mathrm{~m} / \mathrm{s}$ in the domain III $\left(r>x_{2}\right)$, here the convective velocity $V$ is defined as inward for positive sign, and outward for negative sign. The model apparently represents the experiment quite well. In comparison, a SMBI modulation experiment has also been performed for a discharge with a density $\left(n_{\mathrm{e}}=1.9 \times 10^{19} \mathrm{~m}^{-3}\right)$ lower than the critical density $n_{\mathrm{c}}$. In this case, no barriers have been observed and the diffusivity obtained with the same method is $D=0.25 \mathrm{~m}^{2} / \mathrm{s}$ for $r=(28-31) \mathrm{cm}$. In addition, a negative convective velocity has been found as $V=-2.2 \mathrm{~m} / \mathrm{s}$ for $r=(28-31) \mathrm{cm}$ and $V=-4.2 \mathrm{~m} / \mathrm{s}$ for $r=(31-33) \mathrm{cm}$. From the density modulation experiments, it may be concluded that the convective velocity is negative when the density is lower than the threshold and positive when the density exceeds the thresh-

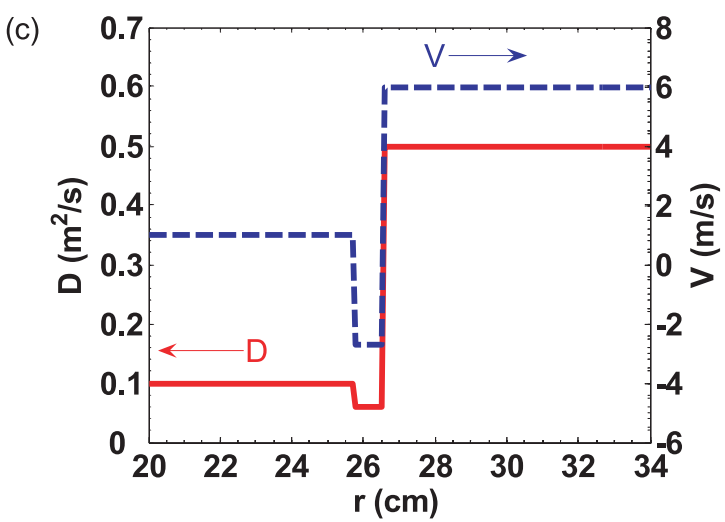

Fig. 2 Profiles of the phase (a) and amplitude (b) of the 1st Fourier harmonic of the modulated density. (c) Profiles of the diffusivity $D$ and convective velocity $V$ for the simulation. 


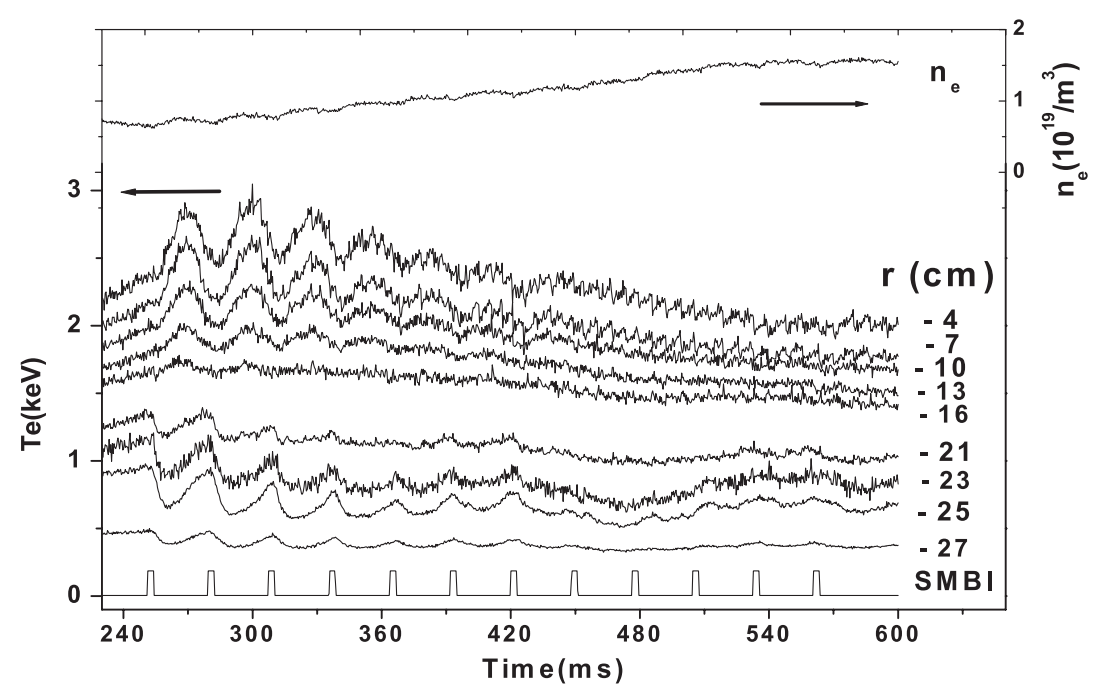

Fig. 3 Temporal evolution of $T_{\mathrm{e}}$ measured by the ECE at different radii.

old, except inside the barrier where the particle convective velocity remains negative. The diffusivity $D$ is rather welllike instead of step-like.

The sign change of the convective velocity can be explained with the TEM/ITG turbulence regimes [10,11]. The density threshold may correlate with the TEM/ITG transition via the collisionality. Generally $V=V_{\mathrm{WARE}}+$ $V_{\nabla q}+V_{\nabla T}$ where $V_{\text {WARE }}$ is the Ware pinch (positive), $V_{\nabla q}$ is the curvature driven pinch (positive), and $V_{\nabla T}$ is the thermodiffusion (positive for ITG and negative for TEM). Usually the Ware pinch is a small effect compared to the turbulence driven effect, except for very flat density profile. For TEM the $V$ can be negative (outward), if the thermodiffusion term is larger than the curvature driven pinch. But it can be also positive (inward) if the thermodiffusion term is less than the curvature driven pinch. It depends mainly the competition between the two terms: the thermodiffsion and the curvature driven pinch. However, the mechanism leading to the pITB formation remains unclear at present. On the other hand, the formation of the barrier may coincide with the TEM/ITG transition. Thus, it is not excluded that the transport barrier is created initially by the discontinuity or jump in the convective velocity during the TEM/ITG transition, or more precisely, by two convective velocities in opposite direction (inward/outward) at the barrier. This speculation needs, of course, further experiments to be confirmed.

\subsection{Non-local transport with SMBI}

Since the first observation of the non-local electron thermal transport on TEXT-U in 1995 [13], a variety of edge cooling techniques [14-16] have been widely used in studying the effect, including impurity injection by laser ablation, ice pellet injection, carbon-based molecule injection, etc. The effect has been observed after supersonic molecular beam being injected the HL-2A tokamak.

Shown in Fig. 3 is the time evolution of electron tem-
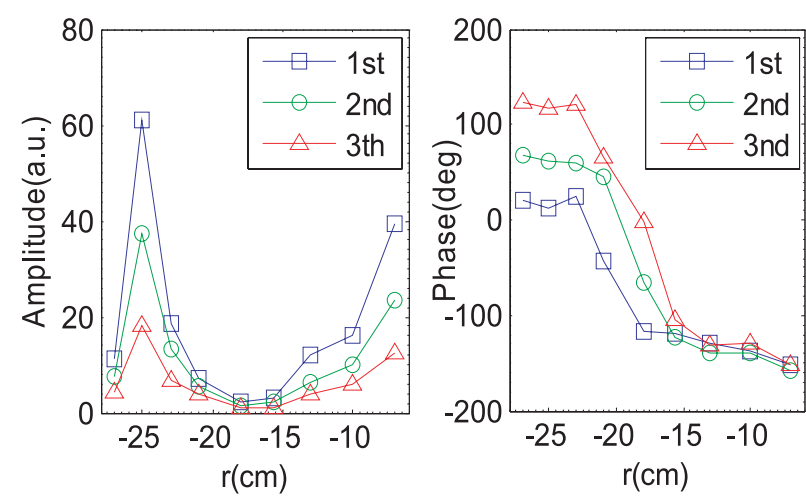

Fig. 4 Amplitudes and phases of the first three harmonics after FFT of the $T_{\mathrm{e}}$ measured by ECE (shot $8337, B_{\mathrm{t}}=1.4 \mathrm{~T}$, $n_{\mathrm{e}}=0.7-1.0 \times 10^{19} \mathrm{~m}^{-3}, I_{\mathrm{p}}=180 \mathrm{k}$.).

perature $T_{\mathrm{e}}$, measured with ECE at different radii during modulated SMBI of $4 \mathrm{~ms}$ duration. The electron temperature sharp dropping in the edge and fast rising in the core after each SMB pulse are clearly shown. In addition, the duration of the core electron temperature rise can be prolonged by adjusting the time separation between two successive SMB pulses. When the line average density increase to about $1.2 \times 10^{19} \mathrm{~m}^{-3}$, the non-local phenomena disappear. Besides, both the thermal radiation and the $\mathrm{H} \alpha$ emission decrease when the non-local effect appears.

The transport properties of the non-local phenomena were analyzed with Fourier transformation of the modulated Te measured with ECE. The results for the modulation of SMBI at $f=10 \mathrm{~Hz}$ are given in Fig. 4. A strong decrease in amplitude and a clear phase jump for all harmonics occur at $r \sim-18 \mathrm{~cm}$, where the reverse of the electron temperature change takes place. The phase change a lot between 23 and $19 \mathrm{~cm}$, indicating the heat pulses propagate much faster than conventional cases. The two peaks in each amplitude profile and the two corresponding troughs in each phase profile are apparent for all harmonics. These 
may indicate that two perturbation sources exist in the regions outside and inside the inversion radius, respectively. The position of the outer initial heat pulse was found to be at $r \sim-25 \mathrm{~cm}$, depending on the deposition location of SMBI. The behavior of the perturbation shows usual propagation features of SMBI cold pulse in this region. The value of $\chi \mathrm{eHP}$ deduced from the Fourier analysis, showing agreement with the result from sawtooth pulse propagation, is in the range of $(2-3) \mathrm{m}^{2} / \mathrm{s}$. In the inner region, the initial perturbation was found to be in the core. The profiles of the amplitude and the phase of the first three harmonics are independent of the SMBI modulation frequency. The steeper profiles around the interface mean a reduction of heat transport and indicate formation of an internal transport barrier in this region. With the good flexibility and easy controllability of the SMBI parameters (gas pressure, modulation frequency, duty cycle, etc.), modulated SMBI has been proven to be an effective tool for studies of the non-local effect. Further investigation on the mechanism of the effect is undertaken on HL-2A.

\subsection{Improved confinement during off-axis ECRH}

The investigation on the suppression of $m=2 / n=1$ tearing mode by off-axis ECRH has been performed. With the instability suppression, obvious increase of the plasma density and stored energy has been observed. A transient improved confinement was obtained after ECRH switchoff in the experiments with the ECRH power in the range of $0.6<P_{\mathrm{ECRH}} / P_{\mathrm{ohm}}<1$. The interesting feature of the confinement after ECRH switch-off motivated us to apply successive ECRH pulses for sustaining MHD-free phase and obtaining a continuous confinement improvement.

The ECRH power must be deposited just around the flux surface where the $m / n=2 / 1$ magnetic islands were located in order to achieve perfect mode stabilization. The frequency and depth of the modulation were about $10 \mathrm{~Hz}$ and $100 \%$, respectively, with a duty cycle of $50 \%$. In this operation mode the maximum power that the gyrotron could deliver was limited to $250 \mathrm{~kW}$. The results from two identical discharges with the ECRH power deposited at (\#8207) and $3 \mathrm{~cm}$ inside (\#8236) the $q=2$ surface, respectively, are shown and compared in Fig. 5. The behaviors of the line averaged density, the central electron temperature (soft x-ray), and the stored energy are the same in the two shots. The amplitude of the $m / n=2 / 1$ magnetic fluctuations begins to decrease after the injection of modulated ECRH at $425 \mathrm{~ms}$ in discharge \#8207. Concurrently with the suppression of the tearing mode the plasma density, the stored energy, and the energy confinement time increase $\sim 80 \%, \sim 50 \%$, and $\sim 40 \%$, respectively, indicating a significant confinement improvement. Thus, the experimental results clearly show that indeed a better suppression may be achieved with such a lower frequency modulated ECRH in the vicinity of the $q=2$ surface. The suppression event is characterized by a feature of the continuous

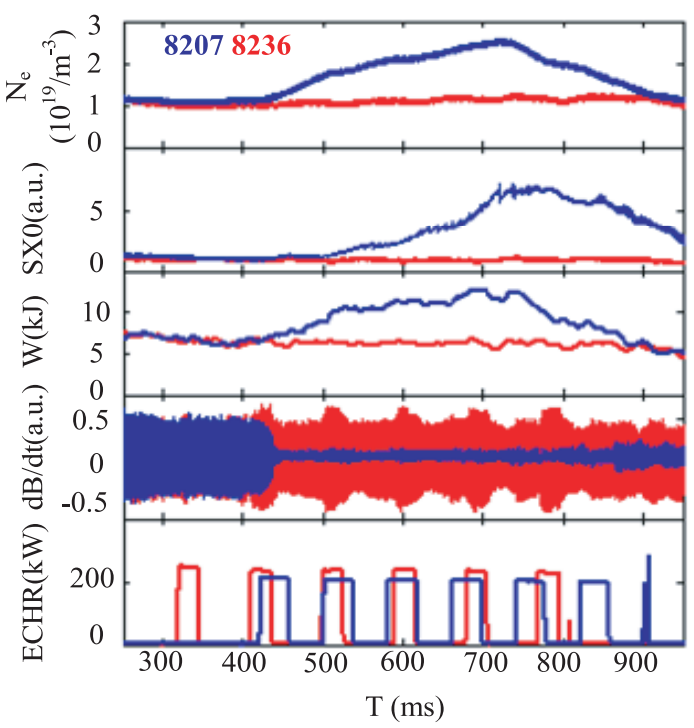

Fig. 5 Time traces of the density, the SXR intensity, the stored energy, the time derivative of the poloidal magnetic field and the power of ECRH deposited at (the blue traces) and inside (the red traces) the $q=2$ surface.

improvement of confinement, i.e. the steady increases of the plasma density, temperature, stored energy and energy confinement time throughout the modulated ECRH period. It should be emphasized that this provides an alternative way to control the $m=2 / n=1$ tearing mode in addition to the continuous ECRH or modulated ECRH with a high frequency in phase with the O-points of the islands.

\section{Conclusions}

A natural particle transport barrier has been evidenced firstly in the ohmic plasmas in HL-2A without any auxiliary heating and external momentum input. The barrier is located around $r / a=0.6-0.7$ with a width of $1-2 \mathrm{~cm}$. A threshold in central line averaged density has been found for the observation of the particle transport barrier with $n_{\mathrm{c}}=2.2 \times 10^{19} \mathrm{~m}^{-3}$. By analysing the propagation of a particle wave, it is found that the diffusivity $D$ is rather well-like than step-like with important reduction inside the barrier. The convection is found to be inward outside of the barrier, and outward inside the barrier.

The non-local phenomena triggered by SMBI have been observed on HL-2A firstly. The core electron temperature $T_{\mathrm{e}}$ rise increases from $18 \%$ to more than $40 \%$ and the duration of the $T_{\mathrm{e}}$ rise could be prolonged by changing the conditions of SMBs injection. Repetitive non-local effect induced by modulated SMBs allows Fourier transformation of the temperature perturbation, yielding detailed investigation of the pulse propagation. The suppression of $m=2 / n=1$ tearing mode has been realized with heating located around $q=2$ surface. The continuous improvement of confinement increase steadily throughout the modulated off-axis ECRH. 


\section{Acknowledgement}

This work was supported by the National Natural Science Foundation of China (Grant No.10335060) and supported partially by the JSPS-CAS Core University Program in the field of 'Plasma and Nuclear Fusion'.

[1] F. Wagner, G. Faussmann, D. Grave et al., Phys. Rev. Lett. 53, 1453 (1984).

[2] M.J. Greenwald, D.A. Gwinn, S. Milora et al., Phys. Rev. Lett. 53, 352 (1984).

[3] C.L. Fiore, J.E. Rice, P.T. Bonoli et al., Phys. Plasmas 8, 2023 (2001).

[4] E.J. Doyle, G.M. Staebler, L. Zeng et al., Plasma Phys. Control. Fusion 42, A237 (2000).

[5] F. Ryter, F. Leuterer, G. Pereverzev, H.U. Fahrbach, J. Stober, W. Suttrop and ASDEX Upgrade Team, Phys. Rev. Let. 86, 2325 (2001).

[6] F. Ryter et al., Plasma Phys. Control. Fusion 48, B453 (2006).
[7] B. Garbet et al., Plasma Phys. Control. Fusion 46, B557 (2004).

[8] A. Jacchia, F. De Luca, S. Cirant, C. Sozzi, G. Bracco, A. Bruschi, P. Buratti, S. Podda and O. Tudisco Nucl. Fusion 42, 1116 (2002).

[9] G.M.D. Hogowij et al., Plasma Phys. Control. Fusion 44, 1155 (2002).

[10] X. Garbet, L. Garzoti, P. Mantica et al., Phys. Rev. Lett. 91, 035001 (2003).

[11] C. Bourdelle, X. Garbet, F. Imbaux et al., Phys. Plasmas 14, 112501 (2007).

[12] S.P. Eury, E. Harauchamps, X.L. Zou et al., Phys. Plasmas 12, 102511 (2005).

[13] J.D. Callen, Phys. Fluids B4, 2142 (1992).

[14] K.W. Gentle et al., Phys. Rev. Lett. 74, 3620 (1995).

[15] G.M.D. Hogeweij et al., Plasma Phys. Control. Fusion 42, 1137 (2000).

[16] X.L. Zou et al., Plasma Phys. Control. Fusion 42, 1067 (2000). 